\title{
Solar quadrupole moment from planetary ephemerides: present state of the art
}

\author{
Sophie Pireaux ${ }^{1}$, E. Myles Standish ${ }^{2}$, Elena V. Pitjeva ${ }^{3}$ \\ and Jean-Pierre Rozelot ${ }^{4}$ \\ ${ }^{1}$ ARTEMIS, Observatoire de la Côte d'Azur, Avenue N. Copernic, F-06130 Grasse, France \\ email: sophie.pireaux@obs-azur.fr \\ ${ }^{2}$ JPL, Caltech, 301-150, Pasadena, CA 91109, USA \\ email: ems@jpl.nasa.gov \\ ${ }^{3}$ Institute of Applied Astronomy of RAS, Nab Kutuzova 10, RU-191187 St Petersburg, Russia \\ email: evp@ipa.nw.ru \\ ${ }^{4}$ GEMINI, Observatoire de la Côte d'Azur, Avenue N. Copernic, F-06130 Grasse, France \\ email: jean-pierre.rozelot@obs-azur.fr
}

\begin{abstract}
We discuss the present state of the art of the solar quadrupole moment from planetary ephemerides.
\end{abstract}

Keywords. gravitation, relativity, ephemerides, Sun: fundamental parameters

Even though the order of magnitude of the Solar quadrupole moment, $J_{2}$, is known to be $10^{-7}$, its precise value is still discussed. Furthermore, stellar equations combined with a differential rotation model, the Theory of Figures of the Sun, as well as inversion techniques applied to helioseismology, are methods which are solar model dependent, i.e., implying solar density and rotation laws. Hence the need for dynamical estimates of the solar quadrupole moment, based on the motion of spacecrafts, celestial bodies or light in the gravitational field of the Sun.

We present an attempt to estimate the solar quadrupole moment via JPL and IAA planetary ephemerides, along with the other ephemeris parameters, through a single step fit to observations. Even though, in principle, it would be possible to extract $J_{2}$ from planetary ephemerides, we observe that it is significantly correlated with other solution parameters (semi-major axis of Mercury or Venus, mass of asteroid ring, ...) in the present ephemerides fitted to the now available observation data. We shall focus on the $J_{2}$ correlations with the Post-Newtonian parameters, characterizing alternative theories of gravitation, according the set of observations considered and the corresponding weight.

The situation shall improve with new data sets (additional VLBI data, additional spacecraft measurements with the ongoing missions, new space missions), the increasing precision in (ranging) observations and the development of new ephemerides. 